\title{
Cluster Based Channel Model and Performance Analysis for MIMO Satellite Formation Flying Communication Systems
}

\author{
Ramoni O. Adeogun \\ School of Engineering and Computer Science \\ Victoria University of Wellington \\ Wellington, New Zealand
}

\begin{abstract}
Satellite formation flying is an essential capability for many space missions that allow several closely spaced smaller satellites to be deployed. Depending on mission requirements, the ground receive station may carry several antennas and receive signal from each of the satellites in order to increase spectral efficiency and Quality of Service (QoS). In this paper, we propose an improved cluster based distributed MIMO channel model based on MIMO models for terrestrial communications in the WINNER/3GPP project for satellite formation flying systems. Monte Carlo simulations were also performed to evaluate the performance of formation flying satellite systems with different configurations using the proposed spatial channel model and comparisons were made with the single satellite-single receive station system using capacity ratio and capacity difference as metrics. Our results show the effects of several factors such as type of formation, number of satellites, number of receive antennas, and SNR on capacity for the single satellite and multi-satellite systems.
\end{abstract}

\section{General Terms:}

MIMO, Satellite Communications, Space propagation

\section{Keywords:}

Clusters, Satellite formation flying, MIMO models, Spatial Channel Model, Capacity

\section{INTRODUCTION}

Satellite formation flying is a significant enabling technology for current and future space-based science missions, astronomical observations and satellite communication systems [1]. Multiple satellites are configured into particular configurations using concepts from computer networking technology forming what is regarded as 'Virtual satellites' with each satellite sharing certain functionalities in the space mission. Formation flying satellites offer several advantages such as improved capacity, flexibility, increased mission reliability and interference mitigation. Satellite formation flying is currently a very hot area of research, however most of the research efforts are geared towards satellite formation design and control [11, 10] leaving other aspects such as propagation channel modelling and performance analysis which are important aspects of any system design open.

Channel modelling for terrestial MIMO wireless communication systems has received tremendous research attention within the last two decades resulting in a number of standardized MIMO models such as COST257 [12] and 3GPP/WINNER II [4] spatial channel models (SCM). These models are based on a combination of both statistical and physical wave propagation channel modelling approaches with small scale and large scale channel parameters fitted to certain distributions based on MIMO measurement campaigns performed in different environments.

A generalized MIMO model was proposed in [2] using deterministic channel modelling approach for satellite formation flying systems. This model was based on the simple summation of sinusoids approach which modelled the non line of sight component as a summation of rays departing the satellites and arriving the ground receiving station with different angle of arrivals, angle of departures, delay of arrivals, and complex amplitudes. This simplified channel modelling approach is not sufficient for realistic transmission environment as it fails to account for the effect of rays that shares common or very closely spaced multipath propagation parameters.

Following the cluster based channel modelling approach for MIMO systems [4] 6] 12], We propose a novel spatial channel model (SCM) for satellite formation flying wireless communication systems and performed Monte Carlo simulations to evaluate its performance using capacity, capacity ratio and capacity difference as metrics. The proposed cluster based channel model accounts for both earth to satellite propagation parameters such as delay of arrival, angle of arrival, angle of departure, delay spread, and power angular spectrum and space communication effects including ionospheric power loss and ionospheric angular deviations.

The remaining parts of this paper are organized as follows. In Section 2, a detailed description of the system model along with the scenarios considered is presented. A discussion of satellite flying formation configurations is presented in Section 3. In Section 4 , We present the proposed cluster based channel model. In Sections 5 and 6, We present expressions for capacity and capacity ratio and difference respectively. Simulations and performance analysis are performed in Section 7. Finally, conclusion is provided in Section 8.

\section{SYSTEM MODEL}

The system considered in this paper is composed of one or more micro-satellites in a linear or circular formation and a ground receiving station with one or more antennas as shown in Figure $1 \square^{1}$ Each satellite in the multisatellite system is assumed to

\footnotetext{
${ }^{1}$ We here make no assumption regarding the satellite orbits. This is to make the channel model a generic model that is application to space
} 
be carrying a single transmitting antenna constituting a Multiple Input Single Output (MISO) or Multiple Input Multiple Output (MIMO) transmission system. The satellite to satellite spacing ranges from 500 meters to $50 \mathrm{~km}$, a distance much smaller than the satellite-to-Earth link. Each satellite in the formation acts as a transmitting antenna. Consider a system with $N$ cooperating transmitting satellite systems in the formation and $M$ receive antennas at the mobile ground receiving station. The received signal at the ground station is given by

$$
\mathbf{y}(\mathbf{t})=\mathbf{H}(\mathbf{t}) \mathbf{x}(\mathbf{t})+\mathbf{w}(\mathbf{t})
$$

where $x(t)=\left[x_{1}(t), x_{2}(t), \cdots, x_{N}\right]^{T} \in \mathbb{C}^{N \times 1}$ is the transmitted signal vector, $y(t)=\left[y_{1}(t), y_{2}(t), \cdots, y_{M}(t)\right]^{T} \in \mathbb{C}^{M \times 1}$ is the receive signal vector, $w(t)$ is an $\mathrm{M}$ dimensional vector of additive Gaussian noise with zero mean and variance $\sigma_{w}^{2}$. The channel impulse response $\mathbf{H}(t)$ is given by

$$
\mathbf{H}(t)=\left[\begin{array}{cccc}
h_{11}(t) & h_{12}(t) & \cdots & h_{1 N}(t) \\
h_{21}(t) & h_{22}(t) & \cdots & h_{2 N}(t) \\
h_{31}(t) & h_{32}(t) & \cdots & h_{3 N}(t) \\
\vdots & \vdots & \ddots & \vdots \\
h_{M 1}(t) & h_{M 2}(t) & \cdots & h_{M N}(t)
\end{array}\right]
$$

$h_{n m}$ is the channel impulse response between the $n$th satellite in the formation and the $m$ th receive antenna at the ground receiving station. We assume that the propagation environment is characterized by a finite number of far field stationary scattering sources. The impulse response between the $n$th satellite in the formation and the $m$ th receive antenna can therefore be modelled as

$$
h_{n m}(t)=\sum_{p=1}^{P} g_{n m}^{(p)}(t) \delta\left(\tau-\tau_{p}\right)
$$

where $g_{n m}^{(p)}(t)$ is the impulse response of the $p^{t h}$ resolvable multipath component (MPC), $P$ is the number of multipath components referred to as Clusters ${ }^{2}, \tau_{p}$ is the delay of the $p$-th cluster.

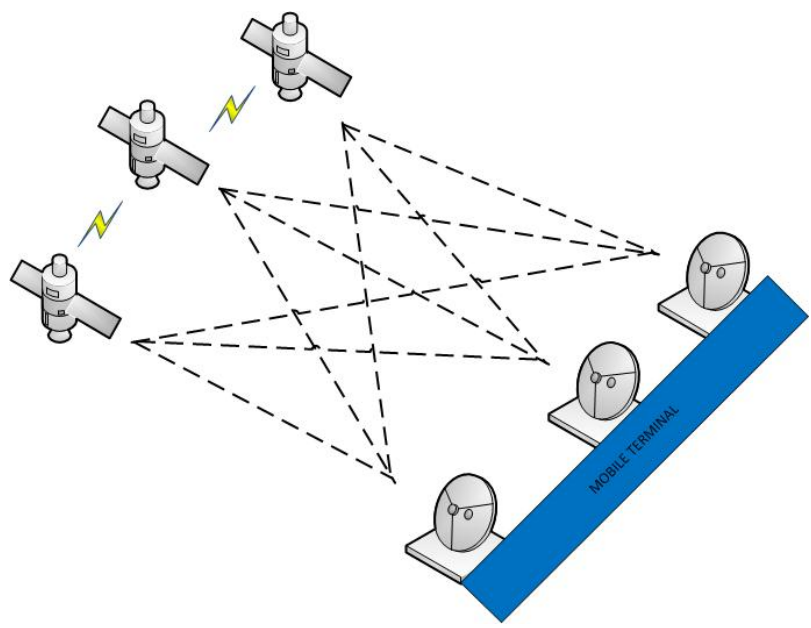

Fig. 1: MIMO satellite Formation Channel. An Illustration of the application of Multiple Input Multiple Output (MIMO) Technique to Satellite Formation Flying

missions in different orbits (e.g. Low Earth Orbit (LEO), Geostationary Orbit (GEO), Medium Earth Orbit (MEO) and so on.)

${ }^{2} \mathrm{~A}$ cluster is defined as a group of unresolvable subrays sharing a common delay of arrival. The clustering assumption has been shown to be valid within several measurement campaign for terrestrial wireless systems.

\section{SATELLITE FORMATION FLYING CONFIGURATIONS}

The configurations of satellite formation considered in this paper are the linear and circular configuration. The linear configuration is considered as a Uniform Linear Array(ULA) with inter-satellite spacing of the order of 1000 times the wavelength. In the circular(round) configuration, the micro-satellites in the formation are considered as being a circular antenna array (see Fig. 2) with the mobile station carrying a linear array of uniformly spaced antennas.

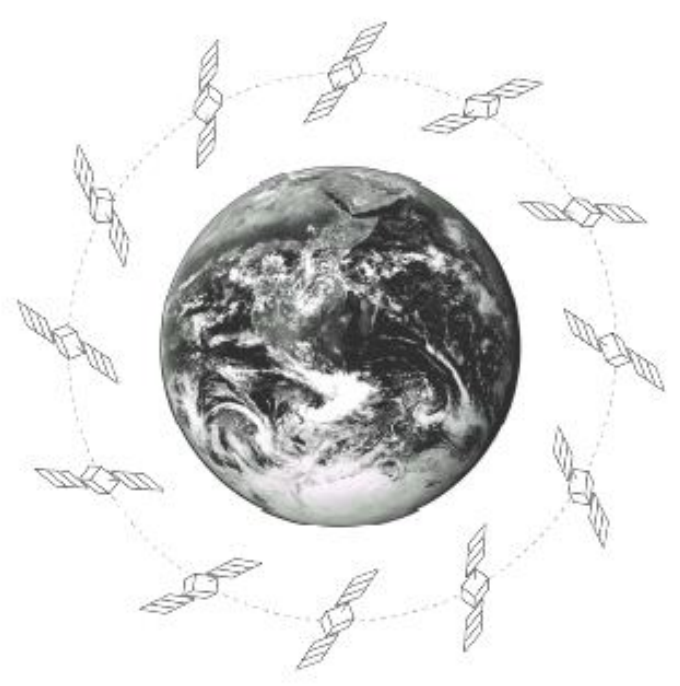

Fig. 2: Circular Satellite Formation 11]

\section{CLUSTER BASED SATELLITE MIMO MODEL}

Recent channel models for terrestrial MIMO wireless communication systems are based on the clustering of rays into multipath components majorly based on the delays of the rays [6, 4]. The WINNER II/3GPP channel model[7] 4] have several specifications for Non line of sight(NLOS) and Line of site (LOS) scenerios. Since typical Satellite channel will always have a LOS component with a particular Ricean K- factor. We relate the 3GPP NLOS MIMO models with typical characteristics of formation flying satellite system. The propose channel model comprises of the NLOS and LOS component and is given as

$$
h_{n m}(t)=\sqrt{\frac{K}{K+1}} h_{n m}^{L O S}(t)+\sqrt{\frac{1}{K+1}} \sum_{p=2}^{P} g_{n m}^{(p)}(t) \delta\left(\tau-\tau_{p}\right)
$$

where $K$ is the Ricean K-factor, $h_{n m}^{L O S}$ is the Line of sight(LOS) component of the channel impulse response between the $n$th satellite and the $m$ th ground receiver antenna. The second term in the RHS of (4) is the non-line-of-sight(NLOS) component of the channel which is modelled as a summation of $P$ clusters, each cluster comprising of $R$ rays. The two terms in the proposed model are given in the following equations

$$
\begin{array}{r}
h_{n m}^{L O S}(t)=\sqrt{P_{p} \exp \left(j \Phi_{p}\right) \cdot G_{R}\left(\theta_{p}\right) \cdot \sigma_{p} \cdot \mathcal{P}_{p} \cdot G_{T}\left(\phi_{p}\right)} . \\
\exp \left(\frac{j 2 \pi}{\lambda}\left(d_{s} \sin \left(\theta_{p}\right)+d_{m} \sin \left(\phi_{p}+\Upsilon_{p}\right)\right)\right) . \\
\exp \left(\frac{-j 2 \pi V_{m}}{\lambda} \cos \left(\vartheta_{v}-\theta_{p}\right)\right)
\end{array}
$$




$$
\begin{array}{r}
g_{n m}^{(p)}(t)=\sqrt{\frac{P_{p}}{R}} \sum_{r=1}^{R} \sqrt{\exp \left(j \Phi_{r p}\right) \cdot G_{R}\left(\theta_{r p}\right) \cdot \sigma_{r p} \cdot \mathcal{P}_{r p} \cdot G_{T}\left(\phi_{r p}\right)} . \\
\exp \left(\frac{j 2 \pi}{\lambda}\left(d_{s} \sin \left(\theta_{r p}\right)+d_{m} \sin \left(\phi_{r p}+\Upsilon_{r p}\right)\right)\right) . \\
\exp \left(\frac{-j 2 \pi V_{m}}{\lambda} \cos \left(\vartheta_{v}-\theta_{r p}\right)\right)
\end{array}
$$

$P_{p}$ is the normalised power of the $p$-th multipath component(MPC), $R$ is the number of rays within each cluster (assumed constant), $\Phi$ is the ionospheric power loss compensation factor for each ray in the clusters, $G_{R}(\theta)$ is the ground receive station array gain for each antenna in the array, $\theta_{r p}$ is the angle of arrival (AOA) of the $r$ th ray in the pth cluster, $\sigma$ is the shadow fading coefficient of the rays, $\mathcal{P}$ is the path loss, $G_{T}(\phi)$ is the satellite transmit antenna response for rays with angle of departure (AOD) $\phi, \lambda$ is the wavelength, $d_{s}$ is the inter-satellite spacing, $\phi_{r p}$ is the angle of departure (AOD) of the $r$ th ray of the $p$ th cluster, $d_{m}$ is the spacing between the antennas on the mobile ground receiving station antenna array, $\phi_{r p}$ is the AOA of the rth ray in the pth cluster, $V_{m}$ is the velocity of the receive station, $\Upsilon$ is the ionospheric angular deviation compensation and $\vartheta$ is the direction of the ground receive station. The MIMO impulse response matrix is obtained from (4) as

$\mathbf{H}(t)=\sqrt{\frac{K}{K+1}} \mathbf{H}^{L O S}(t)+\sqrt{\frac{1}{K+1}} \sum_{p=2}^{P} \mathbf{G}^{(p)}(t) \delta\left(\tau-\tau_{p}\right)$

$\mathbf{H}^{L O S}(t) \in \mathbb{C}^{N \times M}$ is the impulse response matrix for the line of sight component and $\mathbf{G}^{(p)}(t)$ is the non line of sight response matrix for the $p$ th cluster. It can be easily shown from (5) and (6) that

$$
\begin{array}{r}
\mathbf{H}^{L O S}(t)=\sqrt{P_{p} \exp \left(j \Phi_{p}\right) \cdot G_{R}\left(\theta_{p}\right) \cdot \sigma_{p} \cdot \mathcal{P}_{p} \cdot G_{T}\left(\phi_{p}\right)} . \\
\mathbf{a}_{R}\left(\theta_{p}\right) \mathbf{a}_{T}\left(\phi_{p}\right) \exp \left(\frac{-j 2 \pi V_{m}}{\lambda} \cos \left(\vartheta_{v}-\theta_{p}\right)\right)
\end{array}
$$

and

$$
\begin{array}{r}
\mathbf{G}^{(p)}(t)=\sqrt{\frac{P_{p}}{R}} \sum_{r=1}^{R} \sqrt{\exp \left(j \Phi_{r p}\right) \cdot G_{R}\left(\theta_{r p}\right) \cdot \sigma_{r p} \cdot \mathcal{P}_{r p} \cdot G_{T}\left(\phi_{r p}\right)} . \\
\mathbf{a}_{R}\left(\theta_{p}\right) \mathbf{a}_{T}\left(\phi_{p}\right) \exp \left(\frac{-j 2 \pi V_{m}}{\lambda} \cos \left(\vartheta_{v}-\theta_{r p}\right)\right)
\end{array}
$$

where $\mathbf{a}_{R}\left(\theta_{p}\right)$ and $\mathbf{a}_{T}\left(\phi_{p}\right)$ are the array steering vectors at the satellites and ground receiving station respectively. For the uniform linear array and linear satellite formation, the Vandermode structured steering vector is defined as

$$
\mathbf{a}_{R}\left(\theta_{p}\right)=\left[\begin{array}{c}
1 \\
\exp \left(j 2 \pi d_{m} \sin \theta_{p}\right) \\
\exp \left(j 4 \pi d_{m} \sin \theta_{p}\right) \\
\vdots \\
\exp \left(j 2 \pi(N-1) d_{m} \sin \theta_{p}\right)
\end{array}\right]
$$

for the receiving station and

$$
\mathbf{a}_{T}\left(\phi_{p}\right)=\left[\begin{array}{c}
1 \\
\exp \left(j 2 \pi d_{s} \sin \phi_{p}\right) \\
\exp \left(j 4 \pi d_{s} \sin \phi_{p}\right) \\
\vdots \\
\exp \left(j 2 \pi(M-1) d_{s} \sin \phi_{p}\right)
\end{array}\right]
$$

for the linear satellite formation. It should be noted that although the steering vectors are defined for linear arrays, it can be easily extended to the circular array configuration and any other desired satellite and/or receiving antenna geometry.

\section{CHANNEL CAPACITY}

The channel capacity for the single satellite single antenna ground station(SSSAG) is given by the SISO channel capacity as:

$$
C_{S S S A G}=\log _{2}\left(1+\rho|h|^{2}\right)
$$

where $\rho$ is the signal power to noise power ratio(SNR). The capacity for the multi-satellite multiple antenna ground station(MSMA) is given by the generalised MIMO channel capacity without channel state information(CSI) at the transmitter as:

$$
C_{M S M A}=\log _{2}\left(\operatorname{det}\left(I_{M}+\frac{\rho}{N} \mathbf{H H}^{\mathbf{H}}\right)\right)
$$

where $I_{M}$ is the $\mathrm{M}$ by $\mathrm{M}$ identity matrix.[.] ${ }^{H}$ denotes Hermittan transpose, $\rho$ is the signal to noise ratio in $\mathrm{dB}$.

\section{CAPACITY DIFFERENCE AND CAPACITY RATIO}

In order to have a fair comparison of the single satellite and multiple satellite formation systems, we defined the ratio and difference between the multiple antenna capacity and a corresponding number of single antenna systems. The capacity difference is given as:

$$
D_{C A P}=\mathcal{E}\left[C_{M S M A}\right]-n \mathcal{E}\left[C_{S S S A G}\right]
$$

where $n=\min (N, M)$ and $\mathcal{E}[$.$] denotes expectation. The ca-$ pacity ratio is defined as:

$$
R_{C A P}=\frac{\mathcal{E}\left[C_{M S M A}\right]}{n \mathcal{E}\left[C_{S S S A G}\right]}
$$

Equations (14) and (15) can be combined to obtain:

$$
R_{C A P}=\frac{D_{C A P}}{n \mathcal{E}\left[C_{S S S A G}\right]}+1
$$

\section{SIMULATION RESULTS}

In this section, we analysed the performance of linear and circular satellite formation systems in terms of capacity, capacity ratio and capacity difference using the proposed cluster based spatial channel model. The parameters of the satellite formation system used for the simulations in the paper is presented in Table 1 except where otherwise stated. Results for the simulations are averaged over 1000 Monte Carlo simulations. In Figure 3,

Table 1. : Simulation Parameters

\begin{tabular}{|c|c|}
\hline Parameter & Value \\
\hline Ground Receive Station Velocity & $50 \mathrm{~m} / \mathrm{s}$ \\
\hline Inter-Satellite Spacing & $2000 \lambda$ \\
\hline Ground Station antenna spacing & $0.5-1 \lambda$ \\
\hline Satellite Orbit & Geostationary \\
\hline Mobile Speed & $50 \mathrm{Kmph}$ \\
\hline Number of Receive Antenna & $1-8$ \\
\hline Number of Cooperating satellites & $1-8$ \\
\hline Carrier frequency & $14 \mathrm{GHz}$ \\
\hline
\end{tabular}

we present the capacity of linear formation satellite MIMO systems with different number of cooperating satellites and ground receive station antennas against SNR. As shown in the figure, the capacity increases with increasing SNR and as the number of transmitting satellite and/or receive antenna is increasing, the capacity also increases. For instance, with an increase of number of satellites from two to four, an increase of about $10 \mathrm{bps}$ is obtained. This agreed with observations in terrestrial MIMO communication literature and the popular hypothesis of Telatar 
Single Satellite/Multi-Satellite Channel Capacity Vs SNR

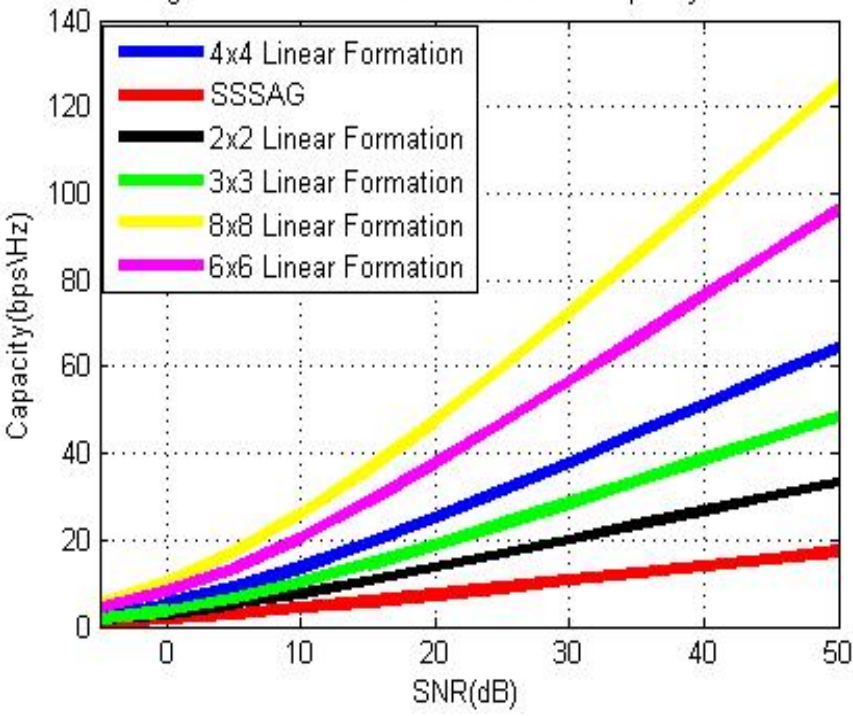

Fig. 3: Capacity Comparison for Linear Formation Satellites with different number of antennas

[3]. In Figure 4, the capacity of linear formation satellite systems with single receive antenna element at the ground station and different numbers of transmitting satellites (corresponding to Multiple Input Single Output (MISO) system) are compared. As shown in the figure, increasing the number of satellites does not offer any significant increase in spectral efficiency. This is expected since theoretical capacity of multiantenna systems only grow with the minimum number of transmit and receive antennas (i.e $C \sim \min (N, M)$ ). Figure 5 present the capacity ratio for different antenna sizes versus the signal to noise ratio (SNR). As can be observed from the figure, the capacity ratio decreases with increasing SNR up till about 10dB and then start to increase almost linearly with further increases in the signal to noise ratio. In Figure 6, we plot the capacity difference versus SNR for different sizes of linear formation satellite systems. As shown in the figure, the capacity difference is minimum for $2 \times 2 \mathrm{MIMO}$ satellite formation flying systems and increases with increasing SNR for all configurations. Figure 7 present the capacity for both linear and circular satellite formations. As shown in the figure the circular satellite formation offers better spectral efficiency compared with the linear formation with a difference of about 10 bps for $3 \times 3$ system and $15 \mathrm{bps}$ for $4 \times 4$ system at a signal to noise ratio (SNR) of $15 \mathrm{~dB}$. These results illustrate the potential of increasing overall spectral efficiency in satellite formation systems by using multiple input multiple output wireless transmission techniques.

\section{CONCLUSION}

A novel cluster based MIMO channel model is proposed in this paper for characterization and performance analysis of satellite formation flying communication systems. The model is based on multipath clustering approach used in standardized MIMO models for terrestrial MIMO Systems. Using the proposed model, we evaluated the performance of linear and circular satellite formation systems using capacity, capacity ratio and capacity difference. Our simulation results showed that capacity of MIMO satellite formation flying systems increases with increasing SNR and number of satellites in the formation, the round formation yielded higher capacity when compared with the linear formation. Future work will analyse the performance of satellite formation flying systems using the proposed channel model and compare with statistical channel models.

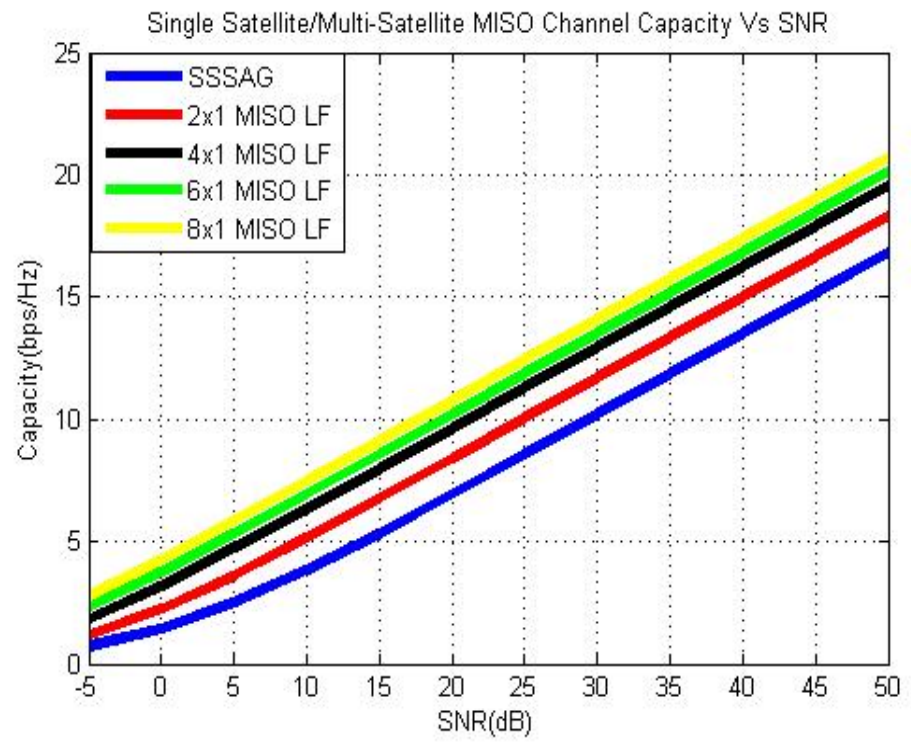

Fig. 4: Capacity Comparison for Linear Formation Satellites with different number of Co-operating Satellites

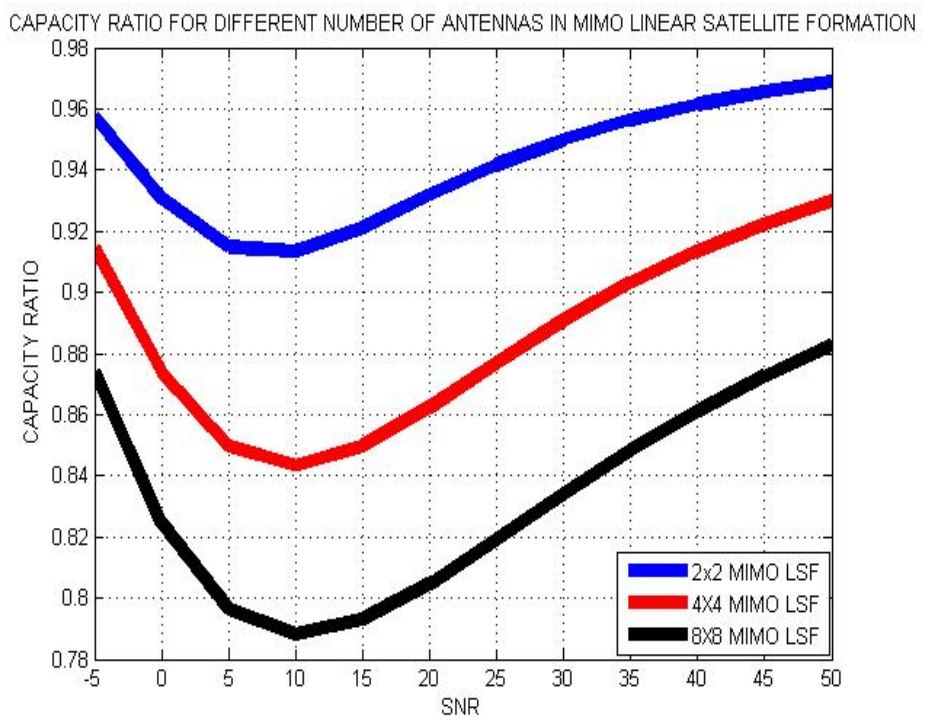

Fig. 5: Capacity Ratio Comparison for MIMO Linear Formation Satellites

\section{REFERENCES}

[1] Online. http://www.esa.int/science/darwin.

[2] L. Zhang, L. Zhu and C. Ju, Generalised MIMO Channel Model and Its Capacity Analysis in Formation Flying Satellite Communication Systems, 6th ICST Conf. on Communications and Networking. China 2011.

[3] I. E. Telatar, Capacity of Multiantenna Gaussian Channels, European Transactions on Telecommunications, vol 10, No.6 pp. 585-595, Nov/Dec 1999

[4] 3GPP TR 25.996, 3rd Generation Partnership Project;technical specification group radio access network;spatial channel model for MIMO simulations(release6), V6.1.0.

[5] A.F. Molish, A Generic Model for MIMO Wireless Propagation Channels in Macro- and Micro Cells, IEEE Trans. 


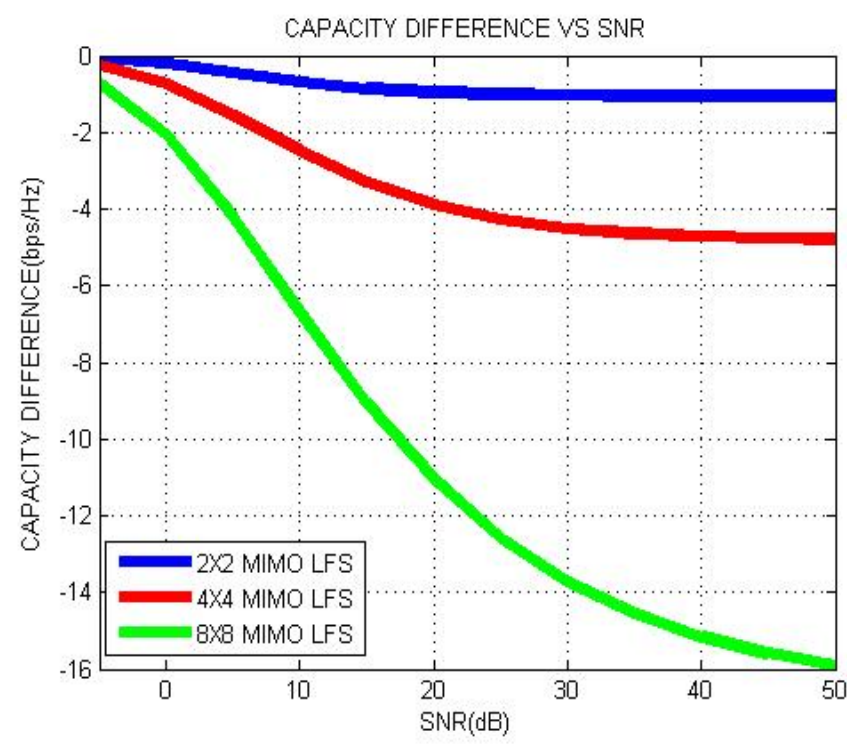

Fig. 6: Capacity Difference for Different Sizes of Linear Formation Flying Systems. Simulation Parameters as shown in 1

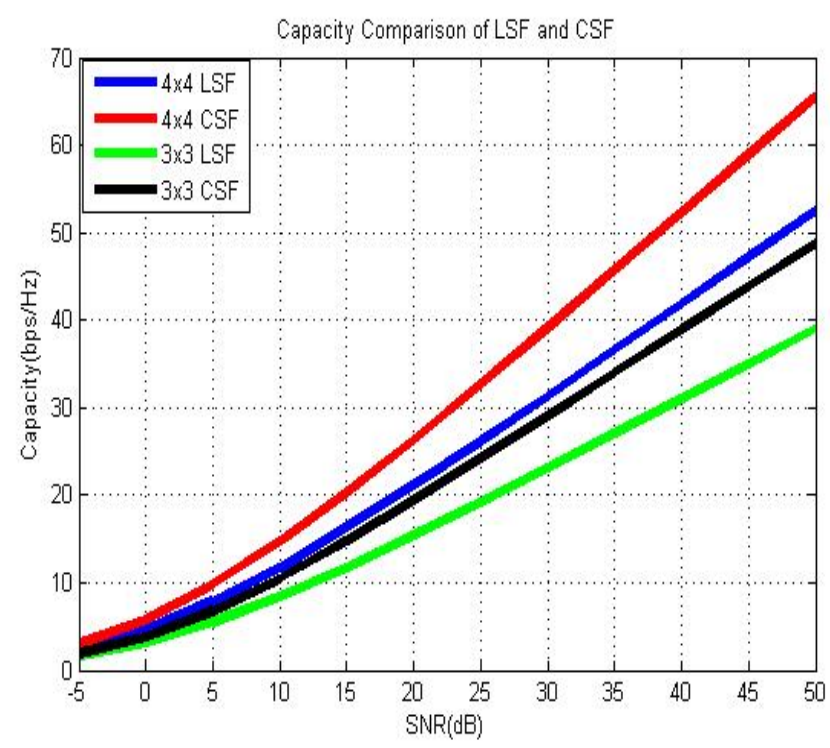

Fig. 7: Capacity Comparison for Linear Formation Satellites and Circular Satellite Formation Configurations on Signal Processing, vol. 52,no. 1,pp 61-71, Jan. 2004.

[6] M. Shafi, M. Zhang and A. L. Moustakas. Polarized MIMO Channels in 3D: Models, Measurements and Mutual Information,IEEE Journal on Selected areas in Communications, volume 24,Issue 3,pp.514-527, March 2006

[7] Hassan El-Sallabi,D. S. Baum, Per Zetterberg,Pekka Kyosti,Terhi Rautiainen, and Christian Schneider. Wideband Spatial Channel Model for MIMO Systems at $5 \mathrm{GHz}$ in Indoor and Outdoor Environments, IEEE 2006

[8] Xiaoyan $\mathrm{Xu}$, Shubo Ren, Jianjun Wu and Haige Xiang. Analysis of Channel Correlation Characteristics in GEO Mobile Satellite Communications IEEE 2011

[9] M. Meshabi and F. Y. Hadaegh. Formation flying control of multiple spacecraft via graphs, matrix inequalities, and switching. AIAA Journal of Guidance, Control, and Dynamics, 24(2):369?377, March-April 2001

[10] Xu Jie Key Techniques of small-satellite formation flight and its development. Aerospace Electronic Warfare 2007, 23(6)

[11] Paolo Massioni,Tamas Keviczky, and Michel Verhaegen. New Approaches to Distributed Control of Satellite Formation Flying. Proc. 3rd Int. Symp. on Formation Flying, Missions and Technologies, Noordwijk , The Netherlands, 2325 April 2008 (ESA SP-654, June 2008)

[12] Wojciech Burakowski and Kenji Leibnitz COST-257 Final Report:An Overview of Activities on Wireless Networks in the European COST-257 Project IFIP PWC'2000 Gdansk, Poland, 14-15 September 2000. 